\title{
Low coronal observations of metric type II associated CMEs by MLSO coronameters
}

\author{
K.-S. Cho ${ }^{1}$, S.-C. Bong ${ }^{1}$, Y.-H. Kim ${ }^{1}$, Y.-J. Moon ${ }^{2}$, M. Dryer ${ }^{3,4}$, A. Shanmugaraju ${ }^{5}$, J. Lee ${ }^{6}$, and Y. D. Park ${ }^{1}$ \\ 1 Korea Astronomy and Space Science Institute, Hwaamdong, Yuseong-Gu, Daejeon, 305-348, Korea \\ e-mail: kscho@kasi.re.kr \\ 2 Dept. of Astronomy \& Space Science, Kyung Hee University, Yongin-shi, Kyungki-do 446-701, Korea \\ 3 NOAA Space Weather Prediction Center, 325 Broadway, Boulder, CO 80305, USA \\ 4 Exploration Physics International, Inc., Huntsville, Alabama, USA \\ School of Physics, Madurai Kamaraj University, Madurai, India \\ ${ }^{6}$ Center for Solar-Terrestrial Research, New Jersey Institute of Technology, Newark, NJ 07102, USA
}

Received 7 November 2007 / Accepted 6 August 2008

\section{ABSTRACT}

\begin{abstract}
Aims. We have investigated the relationship between coronal mass ejections (CMEs) and coronal type II radio bursts by using type II associated CMEs whose low coronal observations by MLSO MK coronameters (1.08-2.85 solar radii for MK4) were available.

Methods. For this we considered all type II burst data at 17:00 UT to 22:00 UT from 1996 to 2003, and then compared them with CME images that were obtained during the same MLSO (Mauna Loa Solar Observatory) observing periods. As a result, we selected 19 type II associated CMEs whose kinematics are well identified. A relationship between CMEs and type IIs has been examined in terms of spatial and temporal closeness without any extrapolation of CME kinematics as well as in terms of CME-streamer interaction. Results. We found that: (1) except one event, all the metric type II events occur simultaneously or after the CME appearance in MK field of view within 30 min, mostly within 10 min after; (2) the distribution of height difference between the CME front and type II formation shows that there are double peaks, one at the CME fronts and the other at about 1 solar radius behind the front; (3) about half of the events (9/19) are identified to have CME-streamer interaction (seven streamer deflection and two overlapping), and the interaction heights are very similar to those of type II formation as well as their interaction times are nearly coincident with those of type II starting; (4) for the other events (10/19), the CME front heights at the starting time of type IIs are comparable to the heights of type II formation.

Conclusions. Our low coronal observations of type II associated CMEs suggest that CME front and/or CME-streamer interaction at CME flank are two main mechanisms to generate type II bursts.
\end{abstract}

Key words. shock waves - Sun: coronal mass ejections (CMEs) - Sun: radio radiation - Sun: corona

\section{Introduction}

Solar metric type II radio bursts showing emission stripes slowly drifting from high to low frequency in dynamic spectrum are regarded to be the signatures of the magneto hydrodynamic (MHD) shocks generated by flares and/or coronal mass ejections (CMEs) (cf. Nelson \& Melrose 1985). The radio emission is due to the shock accelerated electrons that generate plasma oscillations which are subsequently transformed into radio waves escaping the shock vicinity. The drift rate of the burst is about $-0.16 \pm 0.11 \mathrm{MHz} \mathrm{s}^{-1}$, and their life time is in the range of 5 to 15 min (Mann et al. 1996).

There has been a long standing controversy about the origin of metric type II bursts with the two candidate sources of solar flares and CMEs (Wagner \& McQueen 1983; Gosling 1993; Gosling \& Hundhausen 1995; Svestka 1995; Dryer 1996; Gopalswamy et al. 1998; Cliver et al. 1999; Mancuso \& Raymond 2004; Cliver et al. 2005). One view (e.g., Reiner et al. 2001 ) is that a flare-associated coronal shock, detected by the metric type II radio drift, will decay to an MHD wave close to the Sun. This view maintains that the fast CME-type II relation is to be understood as a proxy to the CME-flare relationship because the type II originating in the flare appears to be associated with the CME (see also Dryer 1996). Intimate onset time associations between flares and type II bursts have been reported by several authors (e.g., Harvey et al. 1974; Vřsnak et al. 1995; Cho et al. 2003). However, it is not resolved why the vast majority of flares are not associated with type II bursts. Another view (e.g., Sawyer 1985; Webb \& Howard 1994; Cliver et al. 1999) is that a CME is a special condition for generating type II bursts. In particular, Cliver et al. (1999) insisted that a Moreton wave in the chromosphere, and a type II burst and EIT wave in the low corona are driven by fast CMEs. In addition, Cliver et al. (2005) argued that CMEs are viable drivers for metric type II bursts by reexaminations of six metric type II bursts during November 1997 using LASCO C1 corongraph. All of these six events have been regarded as evidence for non-CME drivers (Reiner et al. 2001; Klein et al. 1999; Warmuth et al. 2001). This view has recently been supported by Gopalswamy et al. (2005) who proposed that the association between type II bursts and CMEs strongly depends on the local Alfvén speed as well as on CME energetics.

Recently, there have been several investigations (e.g., Classen \& Aurass 2002; Lara et al. 2003; Shanmugaraju et al. 2003b; Cho et al. 2005) on the association of metric type IIs with CMEs. Although the emission height of type II bursts is known to be in the range 1.2 to $2.5 R_{\odot}$, these studies were 
based on the extrapolation of CME kinematics observed by $\mathrm{SOHO} / \mathrm{LASCO}$ (Large Angle Spectrographic Coronagraph) due to the lack of low coronal coronagraph images with high temporal resolution. Using 63 metric-type II radio bursts together with SOHO/LASCO and GOES X-ray data, Classen \& Aurass (2002) suggested that type II radio burst excitation may be either due to shocks driven by the CME front, or the internal parts or flanks of the CME. Lara et al. (2003) found that the CMEs associated with type II bursts are more energetic (wider and faster) than regular CMEs. By considering the extrapolated CME height, shock formation heights taking into account type II starting frequencies, and the coronal Alfvén speed profile, Cho et al. (2005) supported the argument of Cliver et al. (1999) that fast CMEs may be an important origin to produce the type II radio bursts. Type II generation ahead of CMEs is demonstrated both theoretically (Dryer et al. 1979; Dryer \& Maxwell 1979) and observationally (Maia et al. 2000). Reiner et al. (2003) suggested a possibility that the type II radio emissions generated by the CME have originated in an enhanced density region of the corona by examining the dynamics of two subsequent CMEs on 2001 January 20. Coronal shocks generated at the CMEs flanks were reported by several studies by using LASCO observation (Sheeley et al. 2000; Vourlidas et al. 2003). By taking into account the geometrical effect between LASCO CMEs and streamers, Mancuso \& Raymond (2004) also showed that type II shocks could be bow/piston driven by the flanks of CMEs. However, since these observations were mainly based on SOHO/LASCO C2 observations, they did not provide us with the direct kinematic information of coronal shocks that associated with type II bursts as well as the interaction between CME flanks and other coronal structures such as helmet streamers.

MK3 and MK4 white light coronameters at Mauna Loa Solar Observatory (MLSO) have low coronal field coverage (1.12-2.42 $R_{\odot}$ for MK3 and 1.08-2.85 $R_{\odot}$ for MK4) with a high time cadence ( $3 \mathrm{~min}$ ) and angular resolutions of about 20 arcsec (MK3) and $12 \operatorname{arcsec}$ (MK4) (Elmore et al. 2003). Their low coronal fields of view with high time cadence allow us to investigate the CME origin of type II bursts by directly examining the kinematics of CMEs at the time of type II bursts. Recently, Cho et al. (2007a) investigated the type II burst associated CME that was observed by MK4 coronamter based on coronal density measurement and concluded that the type II burst was generated at the interface of the CME flank and the streamer. Their result was used to analyze the type II band splitting for estimation of the coronal magnetic field strength (Cho et al. 2007b). Because of these advantages of the MLSO observation, we felt that it might be meaningful to examine the CMEs - type IIs' relationship and the CMEs' interactions with nearby high density streamers, in terms of spatial and temporal closeness. For this, we considered all type II burst data at 17:00 UT to 22:00 UT from 1996 to 2003; we then compared them with MLSO CME images that were available during the same observationally-available period. To our knowledge, such an extensive study has been carried out for the first time to compare low coronal CME kinematics and type II formation in terms of their temporal and spatial closeness without any extrapolation of CME kinematics. For the timing analysis, we compare the start time of a type II with the first appearance time of a CME in Mark 3/4 coronagraph fields of view as well as the interaction time between the CME flank and a pre-existing streamer. For the height comparison, we compare the CME front with the type II formation height estimated from the starting frequency of the radio emission. From these studies, we want to examine which part (front or flank) of a CME is consistent with type II formation height $\left(1.2-2.5 R_{\odot}\right)$. The paper is organized as follows. In Sect. 2, we explain our event selection and data analysis. We present the results of temporal comparison, height comparison, and CME-streamer interaction in Sect. 3. A brief summary and discussion are delivered in Sect. 4.

\section{Data and analysis}

\subsection{Data}

To directly examine the relationship between CMEs and type II bursts, we need white light coronagraph CME images in the low corona covering the type II formation region $\left(1.2-2.5 R_{\odot}\right)$ with high time cadence. Although there was a LASCO C1 coronagraph (1.1-3 $R_{\odot}$ ) from 1996 to 1998 August, its typical time cadence, ranging from several tens of minutes to hours, is not good enough to estimate the kinematics of type II-associated CMEs. Fortunately, Mark K-coronameters (MK3/4) at MLSO have a low coronal field coverage $\left(<3 R_{\odot}\right)$ and high time $(3 \mathrm{~min})$ resolution, thus making it possible to examine the CME kinematics and/or interactions with ambient coronal structures.

The MK3, which had been operated from 1980 to 1999 , had an optical system designed for low scattered light and relatively wide spectral bandpass from $700 \mathrm{~nm}$ to $1080 \mathrm{~nm}$. The MK3 coronal image has a field of view corresponding to $1.12-2.42 R_{\odot}$ with an angular resolution of about $20^{\prime \prime}$ and a temporal cadence of 3 min (Fisher et al. 1981). The MK4 coronameter has been operating from 1998 October to present. The MK4 uses the same optical system as the MK3 instrument, but the low readout noise of the MK4 CCD array improved the signal to noise ratio by a factor of three, allowing the detection of approximately one third more CMEs than with the MK3 coronameter and, also, the detection of events farther away from the Sun. It observes the white-light K-corona over the wavelength range from 700 to $950 \mathrm{~nm}$ with a field of view from 1.08 to $2.85 R_{\odot}$ (Elmore et al. 2003). Its time cadence is the same as that of the MK 3 coronameter. We have used white light CMEs observed by MLSO/MK3 and MK4 K-coronameters from 1996 to 2003 during its daily observing time between $\sim 17: 00$ and $\sim 22: 00$ UT. MK3 and MK 4 coronamaters produce images by rotating a linear CCD array over the FOV, and the measurements at each position angle correspond to different times. We corrected this time difference using the time correction programs provided by the MLSO. For direct determination of CME appearance height and time, we have also used their pre-event subtracted difference images.

We have considered the type II bursts reported in the NGDC/NOAA website ${ }^{1}$ by the spectrographs from the US Air Force Radio Solar Telescope Network (RSTN) and the Culgoora Solar Observatory during the same period. The solar surface locations of the associated flares were taken from National Geographical Data Center (NGDC) ${ }^{2}$ and confirmed from the EUV brightenings in EIT images. In a few cases, their locations were extrapolated from the active region summary compiled by USAF/NOAA.

\subsection{Event selection and analysis}

Our data selection procedure is as follows. First, we have considered 101 type II bursts reported in the NGDC/NOAA website during the MLSO daily observing time (17:00 to 22:00 UT)

\footnotetext{
1 ftp://ftp.ngdc.noaa.gov/STP/SOLAR_DATA/SOLAR_RADIO

2 ftp://ftp.ngdc.noaa.gov/STP/SOLAR_DATA/FLARES
} 
Table 1. Details of type II bursts, flares, and CMEs.

\begin{tabular}{|c|c|c|c|c|c|c|c|c|c|c|c|c|c|}
\hline Type II & & & & & Flare & & & & CME & & & & \\
\hline Date & $\begin{array}{c}T_{\mathrm{II}} \\
\text { (UT) }\end{array}$ & $\begin{array}{c}f_{\mathrm{II}} \\
(\mathrm{MHz})\end{array}$ & $\begin{array}{c}H_{\mathrm{II}} \\
\left(R_{\odot}\right)\end{array}$ & Obs. & $\begin{array}{c}T_{\mathrm{S}} \\
\text { (UT) }\end{array}$ & $\begin{array}{c}T_{\mathrm{P}} \\
\text { (UT) }\end{array}$ & Int & $\begin{array}{l}\text { Loc } \\
\text { (Deg) }\end{array}$ & $\begin{array}{c}\text { PA } \\
\text { (Deg) }\end{array}$ & $\begin{array}{c}T_{\mathrm{fa}} \\
(\mathrm{UT})\end{array}$ & $\begin{array}{l}H_{\mathrm{fa}} \\
\left(R_{\odot}\right)\end{array}$ & $\begin{array}{l}H_{\mathrm{CN}} \\
\left(R_{\odot}\right)\end{array}$ & $\begin{array}{c}V_{\mathrm{C}} \\
\left(\mathrm{km} \mathrm{s}^{-1}\right)\end{array}$ \\
\hline 960505 & $18: 37$ & F50 & 1.50 & PALE & $18: 22$ & $18: 38$ & C31 & S04E93 & 115 & $18: 28: 08$ & 1.2 & 1.4 & 1137 \\
\hline 980830 & $18: 10$ & F80? & 1.32 & SGMR & $18: 00$ & $18: 05$ & M13 & N29W60 & 306 & $17: 12: 47$ & 1.3 & 1.5 & 360 \\
\hline 990120 & 19:14 & F55? & 1.46 & PALE & 19:06 & 20:04 & M52 & N15E74 & 64 & $18: 47: 35$ & 1.8 & 2.6 & 540 \\
\hline 990617 & $17: 33$ & F70 & 1.37 & PALE & $16: 47$ & $17: 30$ & M36 & N19W94 & 285 & $17: 28: 29$ & 1.6 & 1.9 & 527 \\
\hline 990628 & $20: 56$ & F65 & 1.39 & PALE & $20: 55$ & $21: 01$ & $\mathrm{C} 35$ & S25E52 & 130 & $20: 57: 07$ & 1.6 & 1.6 & 1100 \\
\hline 990820 & $18: 39$ & F50 & 1.50 & PALE & $18: 25$ & $18: 29$ & M12 & S23E66 & 104 & $18: 37: 33$ & 2.1 & 2.3 & 441 \\
\hline 991026 & $21: 30$ & H150 & 1.34 & CULG & $21: 09$ & $21: 25$ & M37 & S13W79 & 245 & $21: 16: 44$ & 1.2 & 2.1 & 493 \\
\hline 000205 & $19: 25$ & F75 & 1.34 & PALE & $19: 17$ & $19: 28$ & X12 & N26E52 & 55 & $19: 27: 39$ & 1.3 & 1.3 & 853 \\
\hline 000304 & $18: 18$ & F60? & 1.43 & PALE & $18: 11$ & $18: 32$ & C18 & S16E50 & 135 & $18: 19: 59$ & 1.6 & 1.6 & 331 \\
\hline 000628 & 19:03 & H144 & 1.35 & HOLL & $18: 48$ & 19:10 & C37 & N21W98 & 285 & $18: 44: 34$ & 1.4 & 2.7 & 626 \\
\hline 010730 & $20: 45$ & H180 & 1.28 & PALE & $20: 36$ & $20: 43$ & C60 & S13E61 & 100 & $20: 48: 22$ & 1.6 & 1.6 & 588 \\
\hline 010820 & $19: 24$ & H180 & 1.28 & PALE & 19:19 & $19: 37$ & $\mathrm{C} 23$ & S18E87 & 103 & $19: 23: 31$ & 1.3 & 1.4 & 557 \\
\hline 010912 & $21: 36$ & F41 & 1.60 & PALE & $21: 05$ & $21: 49$ & C96 & S19W50 & 245 & $21: 22: 38$ & 1.4 & 1.8 & 414 \\
\hline 011122 & $20: 27$ & H170 & 1.30 & CULG & $20: 18$ & $20: 36$ & M38 & S24W66 & 242 & $20: 24: 41$ & 1.3 & 1.3 & 1332 \\
\hline 011228 & $19: 59$ & H180 & 1.28 & $\mathrm{CulG}$ & $20: 02$ & $20: 45$ & X34 & S23E102 & 121 & 19:40:22 & 1.5 & 2.2 & 418 \\
\hline 020602 & $20: 40$ & H180 & 1.27 & PALE & $20: 33$ & $20: 44$ & M11 & S30E50 & 135 & $20: 38: 55$ & 1.2 & 1.5 & 700 \\
\hline 021013 & $17: 54$ & H80 & 1.60 & HOLL & $17: 46$ & $17: 57$ & C47 & S07W54 & 255 & $17: 51: 38$ & 1.2 & 1.5 & 623 \\
\hline 021112 & 19:00 & F85? & 1.30 & HOLL & $18: 41$ & $18: 56$ & M29 & S11W76 & 257 & $18: 48: 21$ & 1.1 & 2.1 & 663 \\
\hline 030712 & $19: 07$ & H180 & 1.28 & PALE & $18: 57$ & $19: 06$ & M14 & N16E79 & 75 & $19: 08: 49$ & 1.5 & 1.5 & 580 \\
\hline
\end{tabular}

Note: $T_{\mathrm{II}}, f_{\mathrm{II}}$, and $H_{\mathrm{II}}$ mean start time, start frequency, and start height of the type II burst, respectively. "?" mark denotes ambiguous type II events in identification of the fundamental $(\mathrm{F})$ and harmonic $(\mathrm{H})$ emission bands. $T_{\mathrm{S}}$ and $T_{\mathrm{P}}$ indicate start and peak times of the flare. $T_{\mathrm{fa}}, H_{\mathrm{fa}}$, and $H_{\mathrm{CN}}$ mean first appearance time of the CME, first appearance height of the CME nose, and the nose height measured at type II start time, respectively. $V_{\mathrm{C}}$ means CME speed during type II emissions.

from 1996 to 2003. It is found that 70 type II bursts occurred during the MK3/4 observations and 35 CMEs are identified to have temporal association with the type II bursts. We cannot exclude a possibility that the other half of the events might be related to flare-produced shocks or very faint CMEs which were not detected by the MLSO coronameters. To determine the more definite association between these CMEs and the type II events, we carefully examined all the $35 \mathrm{MK} 3 / 4 \mathrm{CME}$ movies as well as pre-event subtracted difference images. As a result, we selected 19 type II associated CMEs whose expanding structure is sufficiently clear to obtain their height-time data. For these CMEs, we then directly determined their fronts (or leading edges) by visual inspection of the pre-event subtracted images.

Table 1 summarizes the details of the 19 type II associated CMEs together with the information of their related GOES soft X-ray (1-8 $\AA$ ) flares. The first 5 columns give various information of type II events such as type II start time, starting frequency, type II starting height, and their observing stations. Type II starting height is estimated from type II starting frequency by using the Newkirk coronal density model (Newkirk 1961). The next 4 columns represent the associated X-ray flare data such as start/peak time and strength/location. Position angle, first appearance time and height of the CME in the field of view of MK3/4, nose height of the CME at the starting time of type II burst, and the mean CME speed during type II burst emission are given in the last 5 columns. The selected 19 events are found to be mostly limb CMEs whose solar surface longitudinal positions are greater than $50^{\circ}$. Here, the solar surface locations of the CMEs are assumed to be the same as those of the associated solar flares. The first appearance height of the CME fronts (noses) ranges from 1.1 to $2.1 R_{\odot}$, and their heights at the type II burst starting time are estimated to range from 1.3 to $2.7 R_{\odot}$.

\section{Results}

\subsection{Onset time comparison between type IIs and CMEs}

A comparison of the onset time difference among CMEs, flares, and type IIs is a simple way to give us some hints on which one is a driver of the other, or vice versa. There have been several attempts to examine the association between type II burst and CME based on the onset time difference (e.g., Robinson \& Stewart 1985; Lara et al. 2003; Classen \& Aurass 2002; Cho et al. 2003; Shanmugaraju et al. 2003a). The onset time of a CME was often extrapolated from LASCO height time data by assuming that the initial height of the $\mathrm{CME}$ is around $1.1 R_{\odot}$ and that it propagates with a constant speed.

Figure 1 shows the onset time difference between the first appearance of CME in MK3/4 field of view and the type II start. All the type IIs except two events (980830 and 990120) in our sample occurred near the CME appearance time. The time difference mostly lies between -20 and $10 \mathrm{~min}$, and their mean time difference is about $-10 \mathrm{~min}$. We note that such a small time difference is significantly contrasted with previous results (e.g., Classen \& Aurass 2002; Cho et al. 2003) that showed quite scattered distributions with positive and negative time difference using the extrapolation method. These intimate onset associations between the type II bursts and CMEs without any extrapolation may indicate the close association between type II bursts and CMEs as suggested by Cliver et al. (1999).

\subsection{Height comparison between CME front and type II emission}

It is well known that the drifting stripes of metric type II emission are the signature of coronal shock waves. The radio emission frequencies can be converted into emission heights of the 


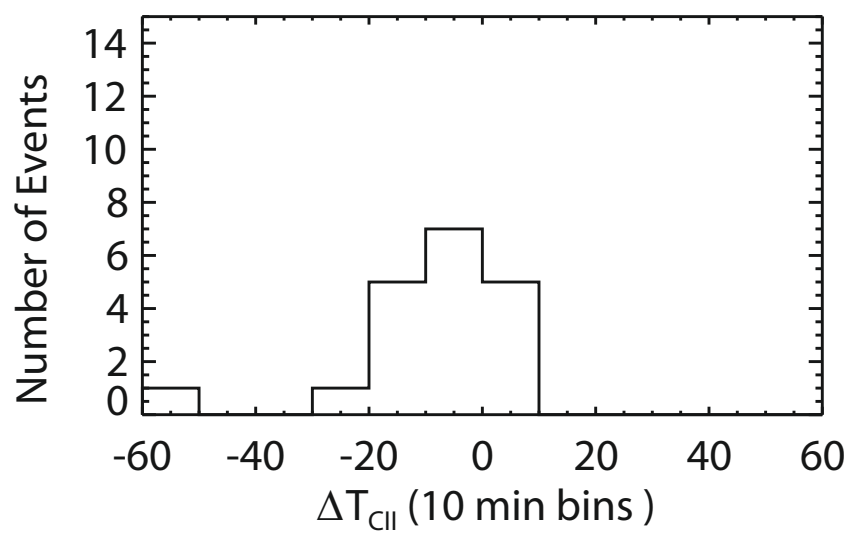

Fig. 1. Histogram of the time difference between CME first appearance in MK3/4 field of view and type II burst start using 10 min bins. $\Delta T_{\mathrm{CII}}$ is equal to CME first appearance time minus type II start time.

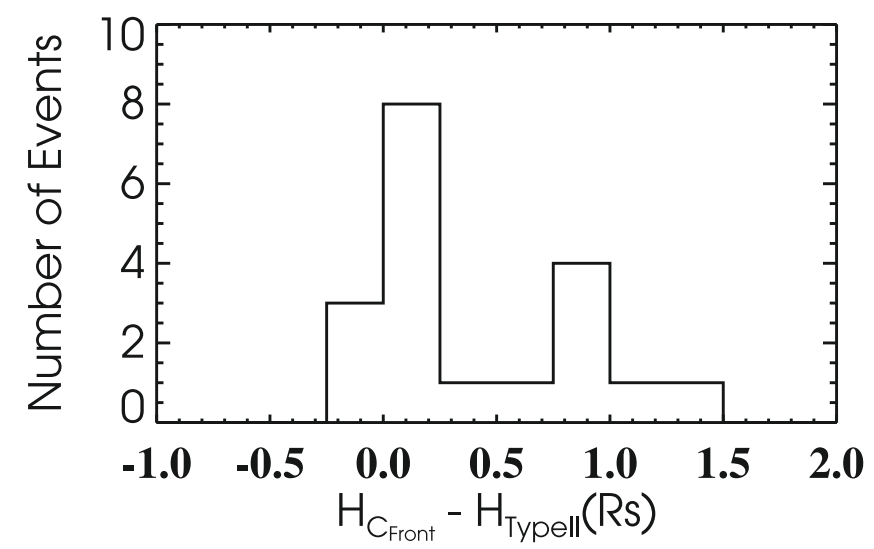

Fig. 2. Distribution of the height difference between CME front and type II burst emission at the starting time of type II burst.

shock by adopting a coronal density model. If a type II burst is generated by the shock ahead of a CME nose, we can simply assume that the CME front would be the source location of type II burst where the radio emission is generated. Thus, we compared the heights of type II shocks estimated from the frequency drift of type II emission with the heights of CME fronts measured by the white-light MLSO MK images.

Figure 2 shows the distribution of height difference between CME front and type II formation at type II starting time. For estimation of the type II formation height, we considered onefold Newkirk coronal density model (Newkirk et al. 1961) and the density measurement from MLSO polarization data (e.g., Cho et al. 2007a). When we compare the heights from the density model to those estimated by using the density measurement along the position angle of the CME nose given in Table 1, we found that the type II heights estimated by using the one-fold Newkirk model are not very different from those derived by density measurements. Their mean and maximum differences are about $0.02 R_{\odot}$ and $0.18 R_{\odot}$, respectively, over all 19 events. Thus we used the density model and estimated the formation height as shown in the 4 th column of Table 1 . The distribution of height difference in the Fig. 2 shows that there are double peaks at the CME front (first group) as well as at about 1 solar radius behind the front (second group). The first group of type II bursts is consistent with our assumption that the type II burst is generated at the CME front. We think of two kinds of possibilities about the second group; the first is that the height difference is due to local density enhancement at the type II formation region; the second

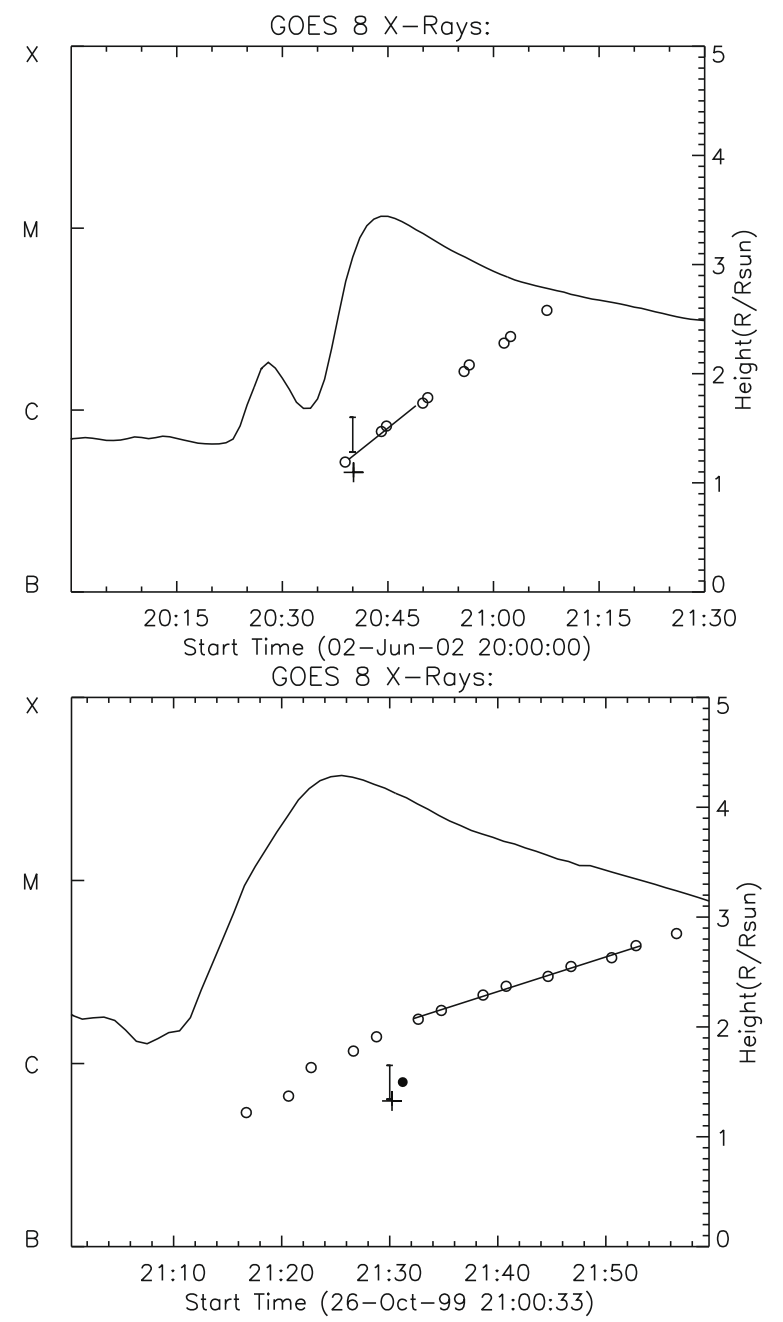

Fig. 3. Height-time plots of CME front (open circle) and type II burst for the CME front associated event (top panel) and for the CME flankassociated event (lower panel). Solid curve represents GOES soft X-ray flux. Solid vertical bar represents the emission height estimated from start frequency of type II burst by using one (bottom of the bar) to four (top of the bar) fold Newkirk density model. Plus symbol denotes the burst height estimated by using MLSO density measurement. The slanted solid lines represent linear fitted heights of CMEs during type II bursts. The filled circle in lower panel denotes the CME flank height interacting with helmet streamer at the type II start time.

possibility is that the type II burst is generated at the CME flank rather than the CME front.

Figure 3 presents the height-time plots of the CME fronts (representative of two groups) that were estimated from measurement of MK4 CME images together with the plot of the type II formation heights based on the Newkirk density model (vertical bar) and density measurement (plus symbol). For type II height from density measurement, we adopted the MLSO density distributions along the selected position angles as denoted by black arrows in Fig. 4. A detailed description of the density measurement from MLSO coronal polarization brightness data can be found in Cho et al. (2007a). For the event on 2002 June 2 (one of the first group) as seen in the upper panel of Fig. 3, the type II burst started just after the first appearance of the CME in the MK4 field of view. In this case, the type II burst was also observed during the impulsive phase of the Xray flare, and its duration is coincident with that of the impulsive phase. Note that the starting height $\left(1.27 R_{\odot}\right)$ of the coronal 

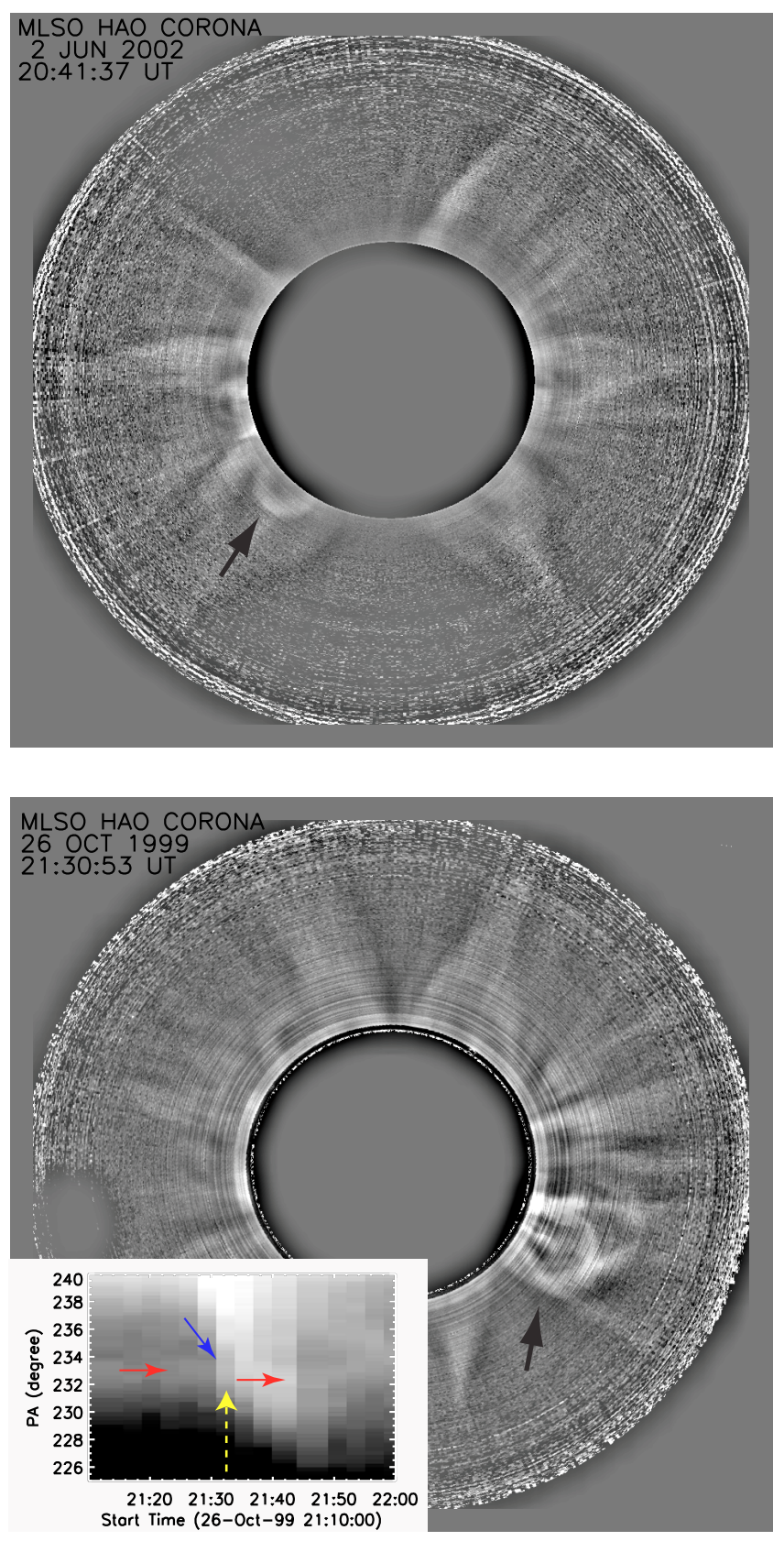

Fig. 4. MK4 image of the CME on 2002 June 2 without a CME-helmet streamer interaction (upper panel) and an enhanced MK4 image of the CME on 1999 October 26 with the helmet interaction (lower panel). The black arrow in the lower panel denotes a place where the interaction between the CME and the streamer exists. Inset image in the lower panel shows the deflection of the streamer by the expanding CME. Slanted (blue) arrows represent the CME propagation and the horizontal (red) arrows indicate the streamer locations before and after the deflection. The interaction time is marked by the vertical yellow arrow.

shock estimated by using the one-fold Newkirk density model is well consistent with the height from MLSO density measurement (plus symbol) as well as the CME nose height (open circle) near the type II starting time. In the figure, the vertical error bar represents the density variation from the one-fold to the fourfold Newkirk density models. The mean speed of the CME during the type II burst is estimated to be $700 \mathrm{~km} \mathrm{~s}^{-1}$. For the 1999 October 26 event (one of the second group) as shown in the lower panel of Fig. 3, the CME front height (open circle) is largely different from the type II shock height estimated by using the
Newkirk model as well as the the height derived by adopting the density measurement (plus symbol) along the CME flank as noted by a black arrow in the lower panel of Fig. 4. However, we note that the type II emission heights are well consistent with the height (filled circle) of CME flank interacting with a helmet streamer, which will be explained in the following section. In this case, type II burst started 15 min after the first CME appearance time and $5 \mathrm{~min}$ after the peak time of the X-ray flare. Mean speed of the CME is about $493 \mathrm{~km} \mathrm{~s}^{-1}$.

One may think of several error sources in shock formation height determination; the determination of the frequency drift rate of type II burst has some errors due to both complex structures of type II emission in the dynamic spectrum and an ambiguity in selection of the frequency drifting edge of the type II bursts. Note that we made one measurement of the lowfrequency edge at the type II starting time. The most significant source of error in the estimation of type II formation height may be local density enhancement. However, the CME height is much deviated from the error bar characterized by the density enhancement from the one-fold to the four-fold Newkirk density model as well as the height from the density measurement. To compensate for such height difference, as shown in the lower panel of Fig. 3, we need the 15-fold Newkirk model, which seems to be too large to be explained by some local density enhancement. As noted before, since the type II heights estimated by using the one-fold Newkirk model is not very different from those derived from density measurements in terms of the type II shock and CME's kinematics' comparison, we have used the one-fold Newkirk model.

Figure 4 shows the two CMEs images presented in Fig. 3: the pre-event subtracted MK4 difference image of 2002 June 2 (upper panel) and the MK4 image of 1999 October 26 (lower panel). These images were taken near the starting time of the type II bursts.

The first CME started to appear at 20:38 UT in the MK4 field of view with the location of the CME front at $1.2 R_{\odot}$. Its position angle is about $135^{\circ}$. Its associated type II started at 20:40 UT and ended at 20:45 UT. The CME front was located at $1.5 R_{\odot}$ at 20:41 UT. As shown in the upper panel of Fig. 3, the CME front height is almost similar to the type II formation height. Thus we think that this type II burst was generated by the CME front, which might be thought of as a laboratory shock tube's piston-driven shock (Dryer 1981) that is formed ahead of an appropriately-fast (i.e., greater than the local fast mode characteristic speed) CME front.

For the second event, the CME was first observed at 21:15 UT, and its front was located at $1.2 R_{\odot}$ in the MK 4 field of view. Its associated type II emission started at 21:30 UT. As noted in the lower panel of Fig. 3, there was a significant height difference between the CME front $\left(2.1 R_{\odot}\right)$ and the type II formation $\left(1.3 R_{\odot}\right)$. We note that there was an interaction between the CME flank and a helmet streamer (denoted by a black arrow in Fig. 4) at a height of $1.5 R_{\odot}$. We found that the CME-streamer interaction is related to a deflection of the streamer as shown in the inset image of Fig. 4, which is the time series image of MLSO brightness data at a given height $\left(1.7 R_{\odot}\right)$. As denoted by red horizontal arrows in the inset image, the position angle of the streamer moves about 1 degree to southward direction just after the sweeping of the CME toward the south-west direction as indicated by a blue slanted arrow. Interestingly, the type II radio emission started just at the deflection time of 21:30 UT (vertical yellow arrow). In addition, this interaction height is comparable with that $\left(1.3 R_{\odot}\right)$ from the estimation of the type II burst (see lower panel of Fig. 3). More detailed morphological features of 
the CME - streamer interaction can be found by watching the corresponding movies available through the MLSO's website ${ }^{3}$. Such temporal and spatial coincidences suggest a possibility that this type II was generated at the interacting region between the CME flank and the helmet streamer.

\subsection{Type II burst and CME-streamer interaction}

It may be meaningful to investigate CME-streamer interactions for other events in terms of their times and heights to look for a possibility that the type II can be generated by the interaction between CMEs and helmet streamers as suggested by Reiner et al. (2003) and reported by van der Holst et al. (2002) and Vourlidas et al. (2003). For this, we took a look at the MLSO CME images and identified that about half of the expanding CMEs (9/19), including the 1999 October 26 event, sweep the helmet streamers aside at the type II starting times. Figure 5 shows the other eight CME events (except the 1999 October 26 event) impinging on nearby streamers. Left image of each panel is enhanced MK4 image and right image is pre-event subtracted image. Faint CMEs are identified from the right images and their sweeping across the streamer can be seen by the left image. As indicated by black arrows on the left image, the CME-streamer interaction heights range from 1.28 to $1.62 R_{\odot}$, which are significantly different from those of the CME front (see 13th column of Table 1). To examine streamer deformation after sweeping of the CME, we investigated the time brightness image at a given height as shown in Fig. 6. From Figs. 5 and 6, we can identify that the streamer deformation is definite for the six events and the other two events (1999 August 20 and 2002 November 12) are not so clear to identify the deformation because of the scattered light in MLSO images. For this reason, we cannot exclude a possibility of the overlapping due to projection effect of the CME for these two events. However, we can see a possibility of the CME-streamer interaction from the MLSO movie ${ }^{3}$. In Fig. 6, there is one event (2001 September 12) that the identification of the streamer position after CME transit is not so easy that we determine the middle position of the reflected streamer. In Table 2, we summarized the CME-streamer interaction with more details such as the interaction time, the interaction height, height difference between the CME nose and the type II formation at the interaction time, and the difference between the interaction height and type II formation height.

Figure 7 shows the distribution of time difference between the CME-streamer interaction and type II starting for these 9 events. As shown in the figure, the starting times of all the type II bursts are well coincident with the interaction time; that is, all the events occurred at the nearly same time within the time range of $\pm 10 \mathrm{~min}$. Their mean absolute time difference is about 2 min.

We want to remind the reader that there are double peaks in the height difference between the CME front and the type II formation in Fig. 2. To examine if these double peaks are related to the CME-streamer interaction, we present in Fig. 8 the height difference for two different groups: 10 events without the interaction (upper panel) and 9 events with the CME-streamer interaction (lower panel). For the first group of events (10/19) without the CME-streamer interaction, the CME front heights at the starting time of type II are comparable to the type II formation heights, as shown in the upper panel of Fig. 8. The mean height difference between the CME front and type II shock is about $0.13 R_{\odot}$ and its root mean square error (rms) is about

http://mlso.hao.ucar.edu/cgi-bin/mlso_acoshome.cgi
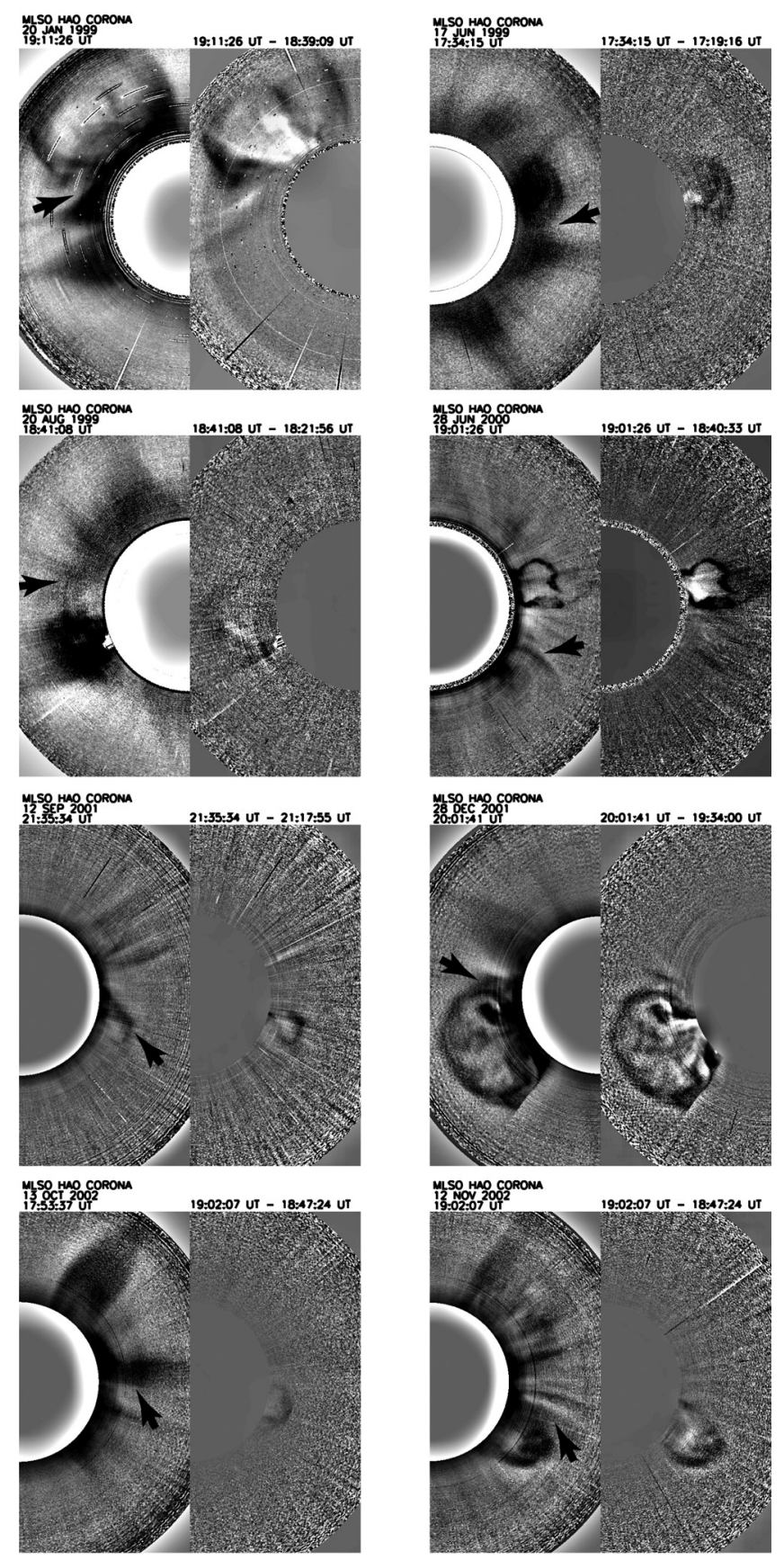

Fig. 5. Enhanced MK4 images and their pre-event subtracted image of the CME-streamer interaction events. The interaction heights determined by eye inspection of the MK4 movies, enhanced MK4 images, and pre-event subtracted images are marked with the black arrows.

$0.13 R_{\odot}$. For the second group of events, their mean height difference between the CME front and type II's formation, as denoted by dotted bars, is about $0.71 R_{\odot}$, and its standard deviation is about $0.44 R_{\odot}$. Except for two events, their height differences are in the range of $0.5 R_{\odot}$ to $1.5 R_{\odot}$. Such large height differences cannot easily be explained by coronal density enhancement, as already noted in the previous section. For two exceptional events whose height differences are relatively small $\left(0.2 R_{\odot}\right.$ for 2001 September 12 event and $-0.1 R_{\odot}$ for 2002 October 13 event), we note that the heights of CME-streamer interaction are nearly similar to the heights of type II formation as well as the CME front. On the other hand, solid bars in the lower panel show the distribution of height difference between 

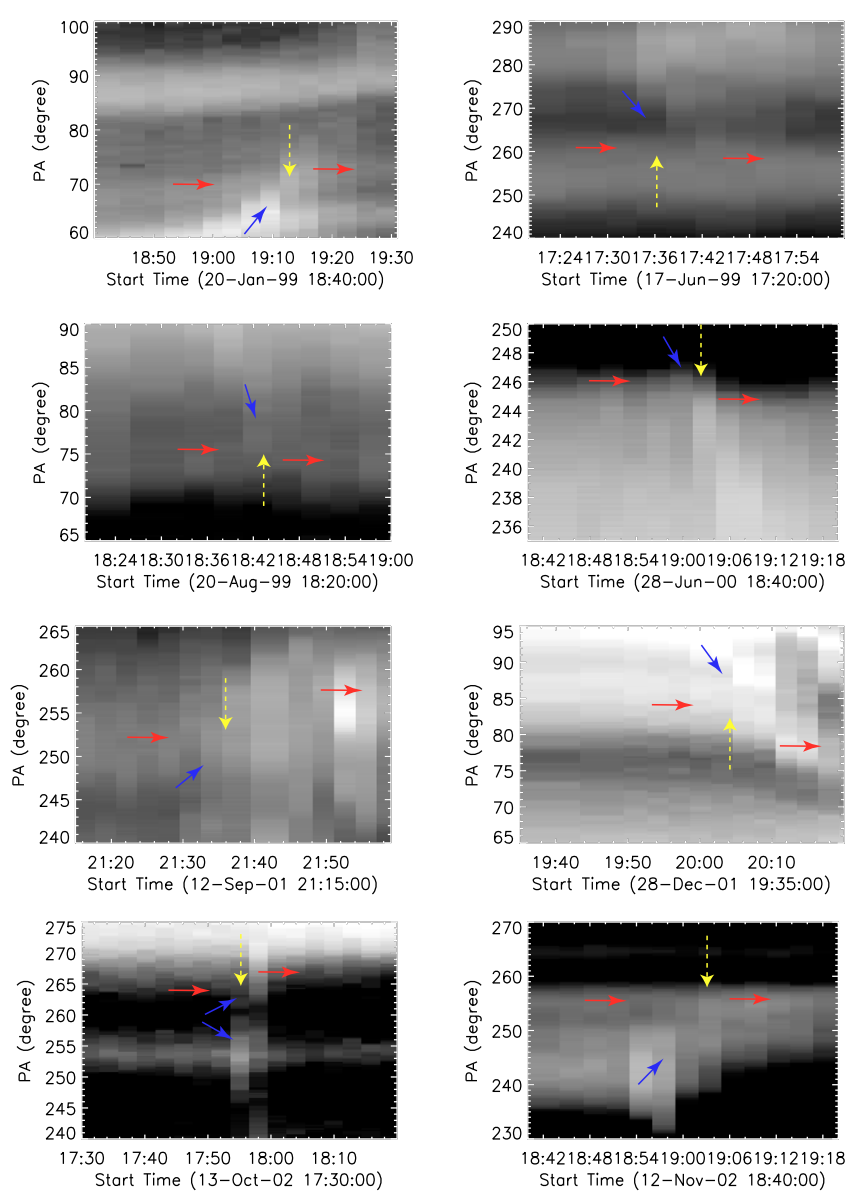

Fig. 6. Time series MLSO brightness data at a given height between 1.4 and $1.8 R_{\odot}$ at which the CME-streamer interaction is well shown. Slanted (blue) arrow indicates the CME propagation and the horizontal (red) arrows denote the streamer location before and after the CME-streamer interaction. The vertical yellow arrows mark the interaction times.

the CME-streamer interaction and the type II formation for the 9 events. We found that the estimated type II formation heights agree very well with those of the interaction within $\pm 0.25 R_{\odot}$ and their absolute mean difference is about $0.1 R_{\odot}$. The differences between the CME-streamer interaction region and the type II formation are estimated to be about $0.01 R_{\odot}$ for 2001 September 12 event and about $0.03 R_{\odot}$ for 2002 October 13 event. Both figures (Fig. 7 and lower panel of Fig. 8) evidently demonstrate that there are very intimate spatial and temporal coincidences between the type II emission and the CME-streamer interaction, strongly supporting the suggestion that the CME-streamer interaction is a potential candidate of type II origin.

For the CMEs whose solar surface locations are closer to $50^{\circ}$, we may expect a projection effects in the height measurement. We thus investigated the longitudinal dependency of the height difference between the CME (nose and flank) and type II burst, and the results are shown in Fig. 9. There may be a weak trend of increasing height difference toward the $50^{\circ}$ longitude but it is not significant.

To get an insight into the physical difference between the groups, we examined the CME speed and height at type II starting time for these two groups in Fig. 10. The triangle symbol indicates the CME of the first group without the streamer interaction, and the filled circle denotes the CME interacting with the streamer. Dotted and solid curves represent the local Alfvén

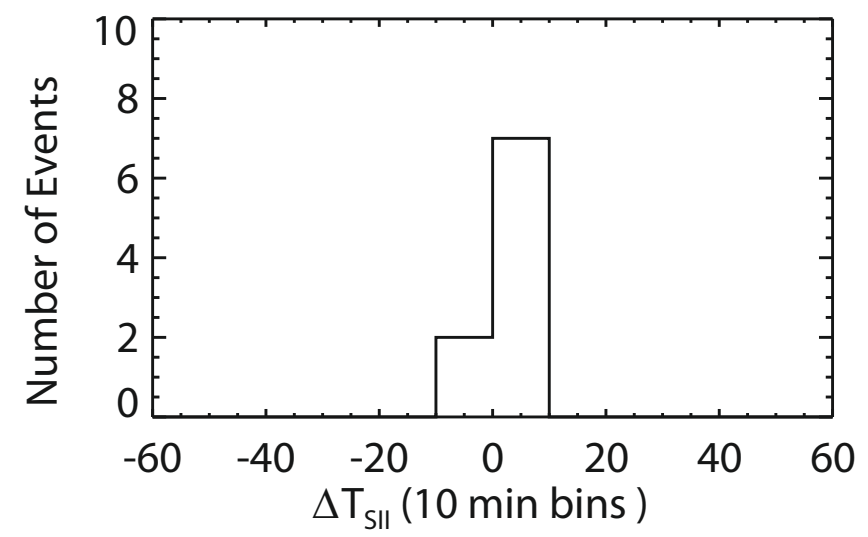

Fig. 7. Histogram of the difference between the CME-streamer interaction time and the type II burst starting time using 10 min bins. $\Delta T_{\mathrm{SII}}$ is equal to CME-streamer interaction time minus type II start time.

speed profiles derived from the active region magnetic field model (Gopalswamy et al. 2001) and quite Sun magnetic field model following Mann et al. (1999), respectively. We adopted here the one-fold Newkirk density model. In general, it is noted that the CMEs in the first group is faster than those of the second group. Their mean speeds are $\left(\sim 753 \mathrm{~km} \mathrm{~s}^{-1}\right)$ and $\left(\sim 527 \mathrm{~km} \mathrm{~s}^{-1}\right)$, respectively. If we take the active region model (dotted curve) as a guide line, the CMEs in the first group are faster than the Alfvén speed, therefore the CMEs could form type II shocks at their noses. On the other hand, CMEs in the second group are on the border line of this Alfvén speed. Supposing that the background density at the streamer is higher and, therefore, that the actual local Alfvén speed is lower than the dashed line, these CMEs could also form shocks at the location of streamer interface. Thus, we speculate that the low Alfvénic region could be a possible-additional reason for having two groups.

\section{Summary and discussion}

There has been the controversy about CME-type II relationship for several decades. Recently, their relationship has been re-inspected by using the extrapolation of CME kinematics in the low corona from SOHO/LASCO C2 and C3 observations. In this study, we have examined the CME - type II burst relationship using the low coronal CME observations covering the metric type II radio emission height without any extrapolation of CME kinematics. For this, we have selected the 19 type II-CME pairs simultaneously observed by radio spectrographs and MLSO MK coronameters with low coronal field coverage (1.1-2.9 solar radii) and high time cadence of $3 \mathrm{~min}$. We then examined the relationship between CMEs (front and CMEstreamer interaction) and type IIs in terms of spatial and temporal closeness. Our main results can be summarized as follows.

(1) Most type II bursts start simultaneously or after the CME first appearance in the MK field of view. All the events, except for one event, occur within $30 \mathrm{~min}$, mostly within $10 \mathrm{~min}$, and their mean difference is about $10 \mathrm{~min}$.

(2) The distribution of height difference between CME front and type II formation shows that there are double peaks, one at the CME front and the other at about 1 solar radius behind the front. This result was illustrated by the examination of the height-time plot of CME front, type II formation height, and MK4 CME images for two events (2002 June 2 and 1999 October 26) in Figs. 3 and 4. While the front of the first 
Table 2. Details of the CME-streamer interaction and the height difference between CME and type II formation. UC means uncertain.

\begin{tabular}{rccccccc}
\hline \hline \multicolumn{2}{c}{} & \multicolumn{1}{c}{ Streamer interaction } & & & \\
\hline Date & $\begin{array}{c}\text { CME Sweeping } \\
\text { Yes/No }\end{array}$ & $\begin{array}{c}\text { Streamer deflection } \\
\text { Yes/No }\end{array}$ & $\begin{array}{c}\text { PA } \\
(\mathrm{Deg})\end{array}$ & $\begin{array}{c}T_{\mathrm{I}} \\
(\mathrm{UT})\end{array}$ & $\begin{array}{c}H_{\mathrm{I}} \\
\left(R_{\odot}\right)\end{array}$ & $\begin{array}{c}H_{\mathrm{CN}}-H_{\mathrm{II}} \\
\left(R_{\odot}\right)\end{array}$ & $\begin{array}{c}H_{\mathrm{I}}-H_{\mathrm{II}} \\
\left(R_{\odot}\right)\end{array}$ \\
\hline 960505 & No & No & - & - & - & -0.10 & - \\
980830 & No & No & - & - & - & 0.18 & - \\
990120 & Yes & Yes & 70 & $19: 13: 27$ & 1.40 & 1.14 & -0.06 \\
990617 & Yes & Yes & 260 & $17: 36: 10$ & 1.47 & 0.53 & 0.1 \\
990628 & No & No & - & - & - & 0.21 & - \\
990820 & Yes & UC & 75 & $18: 43: 23$ & 1.62 & 0.80 & 0.12 \\
991026 & Yes & Yes & 233 & $21: 32: 45$ & 1.50 & 0.76 & 0.16 \\
000205 & No & No & - & - & - & -0.04 & - \\
000304 & No & No & - & - & - & 0.17 & - \\
000628 & Yes & Yes & 245 & $19: 02: 06$ & 1.50 & 1.35 & 0.15 \\
010730 & No & No & - & - & - & 0.32 & - \\
010820 & No & No & - & - & - & 0.12 & - \\
010912 & Yes & Yes & 255 & $21: 36: 31$ & 1.61 & 0.20 & 0.01 \\
011122 & No & No & - & - & - & 0.00 & - \\
011228 & Yes & Yes & 85 & $20: 03: 54$ & 1.43 & 0.92 & 0.15 \\
020602 & No & No & - & - & - & 0.22 & - \\
021013 & Yes & Yes & 265 & $17: 55: 14$ & 1.35 & 0.18 & 0.03 \\
021112 & Yes & UC & 255 & $19: 03: 51$ & 1.28 & 0.80 & -0.02 \\
030712 & No & No & - & - & - & 0.20 & - \\
\hline
\end{tabular}
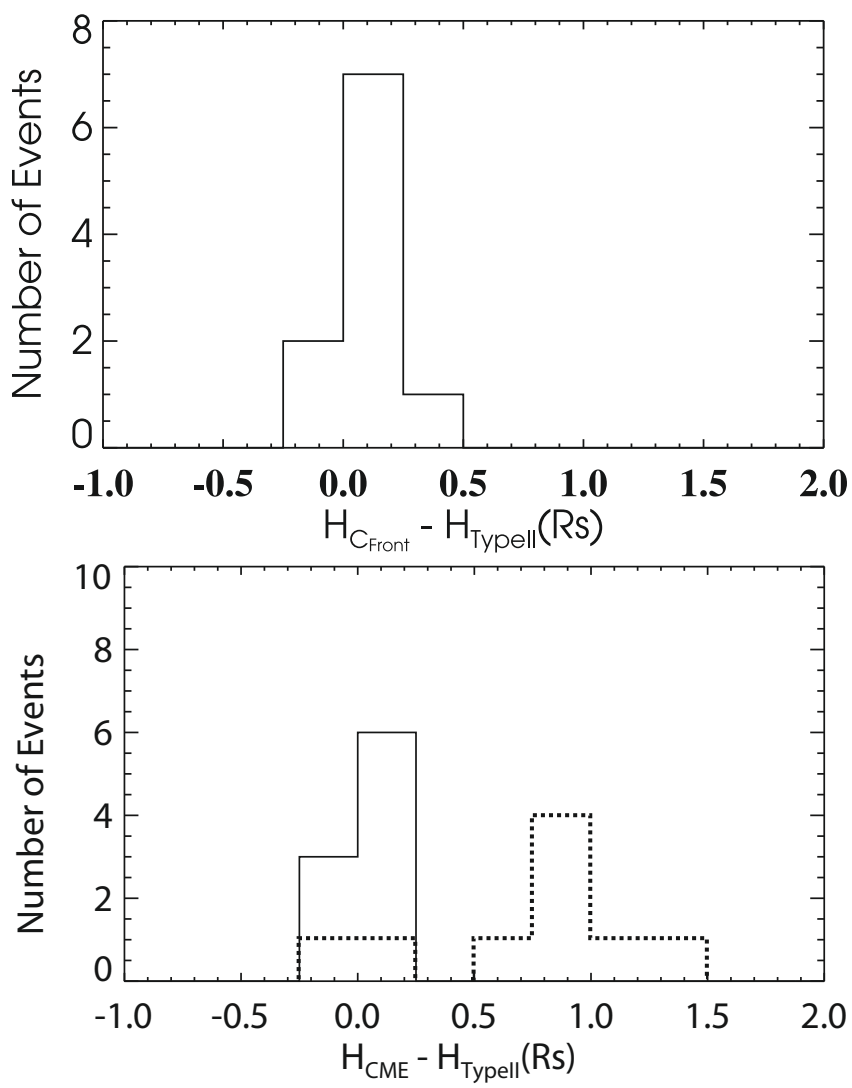

Fig. 8. Histograms of the height difference between the CME front and type II formation for the events without the CME-streamer interaction (upper panel) and with the CME-streamer interaction (dotted bars in lower panel). Solid bars in lower panel denote the difference between the height of CME-streamer interaction and the height of type II burst emission.

CME is well consistent with the type II emission height, the front of the second CME is significantly different from the type II emission height, which cannot be explained by local density enhancements.

(3) From the investigation of MK3/4 images of 19 type II associated CMEs, we identified that about half of the events $(9 / 19)$ have the CME-streamer interaction. It is found that seven events show the streamer deflection after impingement of the CME. We note that their type II formation heights are very similar to the interaction height between the CME flank and the high density streamers, and the emission times are nearly coincident with the interaction times. Thus, these events seem to be good examples of type II events generated by the interaction between the CME flank and the high density streamer.

(4) It turned out that the CMEs without interaction $\left(\sim 753 \mathrm{~km} \mathrm{~s}^{-1}\right)$ are faster than the streamer interacting CMEs $\left(\sim 527 \mathrm{~km} \mathrm{~s}^{-1}\right)$ as well as the local Alfvén speed $\left(\sim 400 \mathrm{~km} \mathrm{~s}^{-1}\right)$. On the other hand, the CMEs interacting with the streamer are located on the border line of the Alfvén speed. Supposing that actual local Alfvén speed at the streamer could be lower because of higher density of the streamer, these CMEs could also form shocks at the streamer interface's location. This is well consistent with recent theoretical investigation of the type II burst onset by Lin et al. (2006) who demonstrate that the onset of type II bursts depends on the local Alfvén speed (e.g. see their Fig. 3).

The above results strongly support the suggestion that there are two kinds of type II generation by CMEs. Thus we speculate that the local density and Alfvén speed could be the reason for having two groups: the first kind of type II is generated ahead of the CME front and the second is generated at the CME flank. In the case of the first type of events, we found very close time and height association between the CME front and the type II burst, supporting the coronal shock generation near the CME front. These results are consistent with those of Maia et al. (2000) who showed a correspondence in temporal and spatial association between the radio type II weak sources obtained with the Nancay 


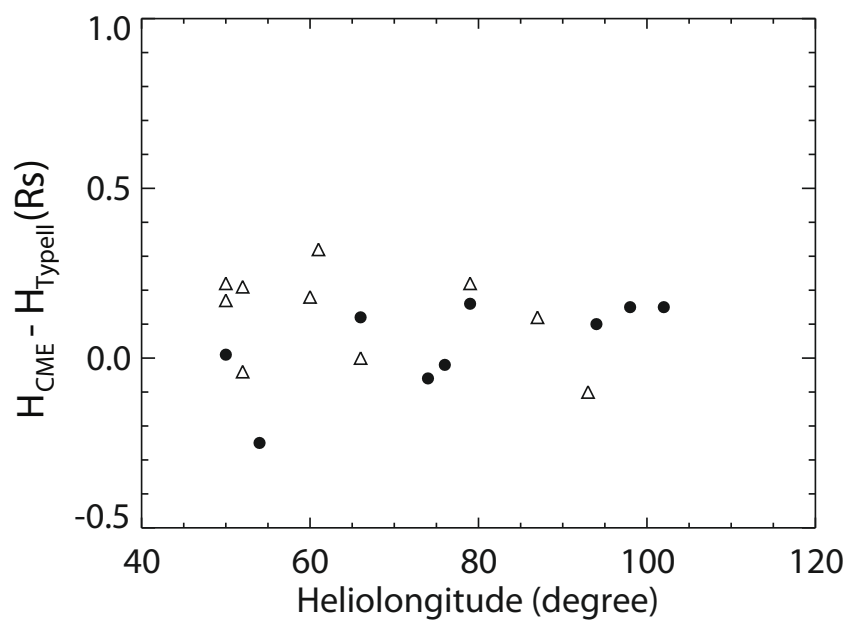

Fig. 9. Longitudinal dependency of the height difference between the CME (nose and flank) and type II burst for CME nose associated events (triangle) and the CME-streamer interacting events (filled circle).

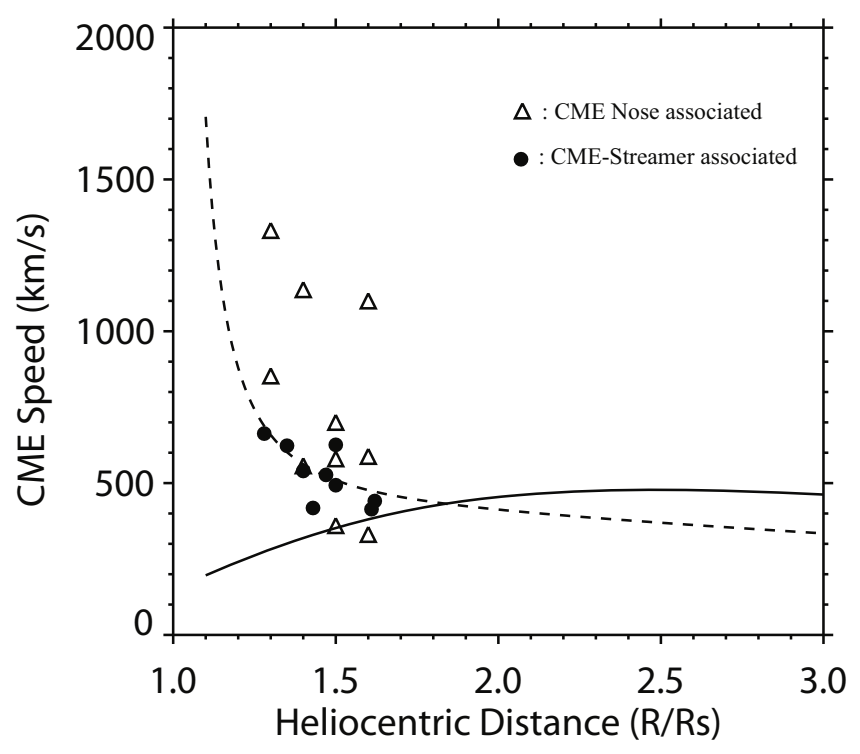

Fig. 10. CME speed vs. CME nose (triangle) and streamer interacting flank (filled circle) heights. Curves denote the Alfvén speed derived from the quiet solar magnetic field model (solid) and active region magnetic field model (dotted) using the one-fold Newkirk density model.

radioheliograph and the front of CMEs seen by LASCO C1. For the second type of the events, our analysis showed that there is a temporal and spatial closeness between CME-streamer interaction and type II burst. These facts are consistent with the arguments of Reiner et al. (2003) and Cho et al. (2007a) that the type II radio emissions associated with $\mathrm{CME}$ events must have originated in high-density structure in the corona. Interestingly, our results are also consistent with the argument of Mancuso \& Raymond (2004) who insisted that type II shocks could be bow/piston driven by the top or the flanks of CMEs, if appropriate geometrical considerations were taken into account.

A cartoon in Fig. 11 represents a summary of our results on the two possible scenarios for generation of type II bursts related with CMEs. One view concerns type II generation at (or near) the CME front, when a fast CME passes through the corona. In this case, the type II burst emission height is comparable to the CME front height, as shown in the upper panel of Fig. 8. This scenario is also consistent with the theoretical

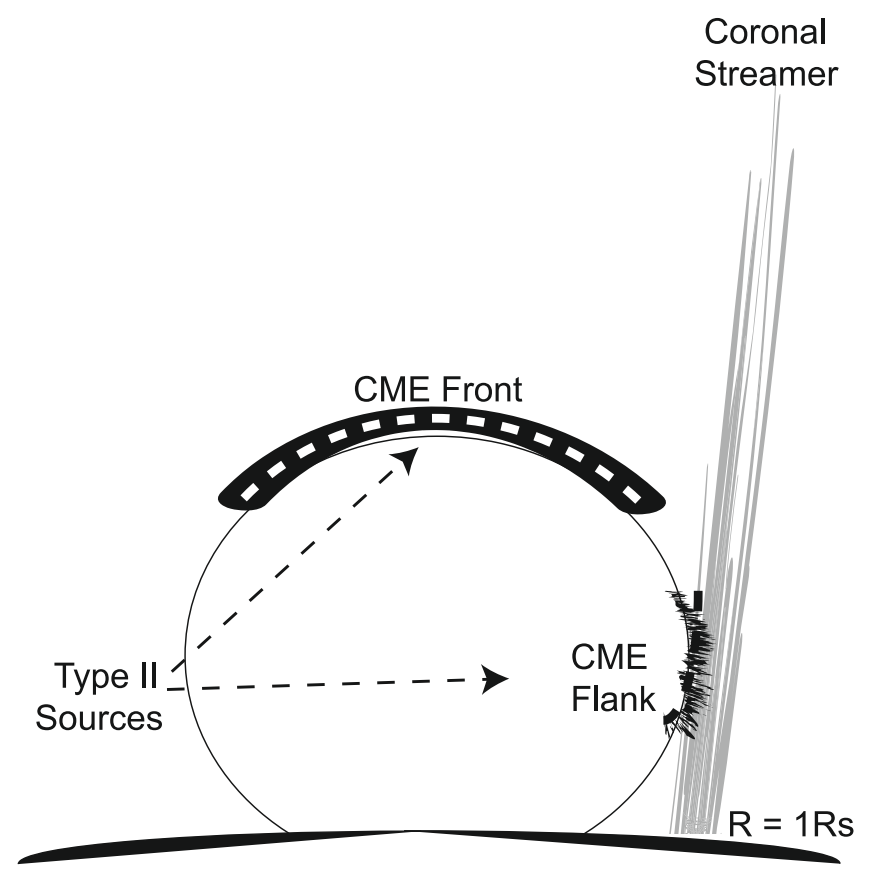

Fig. 11. A cartoon showing two main origins of type II emissions by CMEs.

2D MHD simulations of Dryer et al. (1979), Dryer \& Maxwell (1979), and many others since that time. However, the physical details of the initialization process (pressure pulse, thermal heating, flux emergence, reconnection, field line motion, etc.) is still unclear. The other scenario is that the type II burst starts to appear at the flanks of the CMEs when the flanks interact with a high density streamer (low Alfvénic region) even though the CME is not so fast. Type II emission heights are relatively lower than the CME fronts but comparable to the CME-streamer interaction height. It means that coronal density enhancement regions such as helmet streamers give a good environment to generate type II radio bursts. Our low coronal observations of type II associated CMEs suggest that the CME front and/or the CME-streamer interaction are two main mechanisms to generate type II radio bursts.

Acknowledgements. We thank the anonymous referee for comments that helped to improve the manuscript. The Mauna Loa Solar Observatory (MLSO) is operated by the High Altitude Observatory (HAO), a division of the National Center for Atmospheric Research (NCAR), which is sponsored by the National Science Foundation (NSF). We greatly thank J.C. Chae and B. Joshi for their useful comments. This work has been supported by the "Development of Korean Space Weather Center", a project of KASI, and the KASI basic research fund. M.D. was supported by NASA's Living With a Star (LSW) Targeted Research and Development Program via NASA Grant NAG-12527; he also thanks the NOAA Space Weather Prediction Center for providing emeritus hospitality. YJM was supported by the Korea Research Foundation (KRF-2005-070-C00059) of the Korean government.

\section{References}

Cho, K.-S., Kim, K.-S., Moon, Y.-J., \& Dryer, M. 2003, Sol. Phys., 212, 151 Cho, K.-S., Moon, Y.-J., Dryer, M., et al. 2005, J. Geophys. Res., 110, A12101 Cho, K.-S., Lee, J., Moon, Y.-J., et al. 2007a, A\&A, 461, 1121

Cho, K.-S., Lee, J., Gary, D.-E., Moon, Y.-J., \& Park, Y.-D. 2007b, ApJ, 665, 799

Classen, H. T., \& Aurass, H. 2002, A\&A, 384, 1098

Cliver, E. W., Webb, D. F., \& Howard, R. A. 1999, Sol. Phys., 187, 89

Cliver, E. W., Nitta, N. V., Thompson, B. J., \& Zhang, J. 2005, Sol. Phys., 225, 105 
Dryer, M. 1981, in Solar Wind Four, ed. H. Rosenbauer, Max-Planck Inst. Fur Aeronomie Rept. MPAE-W-100-81-31, 199

Dryer, M. 1996, Sol. Phys., 169, 421

Dryer, M., \& Maxwell, A. 1979, ApJ, 231, 945

Dryer, M., Wu, S. T., Steinolfson, R. S., \& Wilson, R. M. 1979, ApJ, 227, 1059

Elmore, D. F., Burkepile, J. T., Darnell, J. A., Lecinski, A. R., \& Stanger, A. L. 2003, Proc. SPIE, 4843

Fisher, R. R., Lee, R. H., MacQueen, R. M., \& Poland, A. I. 1981, Appl. Opt., 20,6

Gopalswamy, N., Kaiser, M. L., Lepping, R. P., et al. 1998, J. Geophys. Res., 103,307

Gopalswamy, N., Lara, A., Kaiser, M. L., \& Bougeret, J.-L. 2001, J. Geophys. Res., 106, 25261

Gopalswamy, N., Aguilar-Rodriguez, E., Yashiro, S., et al. 2005 , J. Geophys. Res., 110, A12

Gosling, J. T. 1993, J. Geophys. Res., 98, 18937

Gosling, J. T., \& Hundhausen, A. J. 1995, Sol. Phys., 160, 57

Harvey, K. L., Martin, S. F., \& Riddle, A. C. 1974, Sol. Phys., 36, 151

Klein, K.-L., Khan, J. I., Vilmer, N., Delouis, J.-M., \& Aurass, H. 1999, A\&A, 346, L53

Lara, A., Gopalswamy, N., Nunes, S., Munoz, G., \& Yashiro, S. 2003, Geophys. Res. Lett., 30, 8016

Lin, J., Mancuso, S., \& Vourlidas, A. 2006, ApJ, 649, L1110

Mancuso, S., \& Raymond, J. C. 2004, A\&A, 413, 363

Mann, G., Klassen, A., Classen, H.-T., et al. 1996, A\&AS, 119, 489

Mann, G., Aurass, H., Klassen, A., Estel, C., \& Thompson, B. J. 1999, in Proc. of 8th SOHO Workshop (Paris, Eur. Space Agency), ESA SP-446, 447
Maia, D., Pick, M., Vourlidas, A., \& Howard, R. 2000, ApJ, 528, L49

Nelson, G. J., \& Melrose, D. B. 1985, in Solar Radio Physics, ed. D. J. McLean, \& N. R. Labrum (New York: Cambridge), 333

Newkirk, G. Jr. 1961, ApJ, 133, 983

Robinson, R. D., \& Stewart, R. T. 1985, Sol. Phys., 97, 145

Reiner, M. J., Kaiser, M. L., Gopalswamy, N., et al. 2001, J. Geophys. Res., 106, 25279

Reiner, M. J., Vourlidas, A., Cyr, O. C. St., et al. 2003, ApJ, 590, 533

Sawyer, C. S. 1985, Sol. Phys., 98, 369

Shanmugaraju, A., Moon, Y.-J., Dryer, M., \& Umapathy, S. 2003a, Sol. Phys., 215,161

Shanmugaraju, A., Moon, Y.-J., Dryer, M., \& Umapathy, S. 2003b, Sol. Phys., 217,301

Sheeley, N. R. Jr, Hakala, W. N., \& Wang, Y.-M. 2000, J. Geophys. Res., 105, 5081

Svestka, Z. 1995, Sol. Phys., 160, 53

Vourlidas, A., Wu, S. T., Wang, A. H., Subramanian, P., \& Howard, R. A. 2003, ApJ, 598, 1392

Van der Holst, B., Van Driel-Gesztelyi, L., \& Poedts, S. 2002, in Proc. 10th European Sol. Phys. Meeting, ed. A. Wilson, ESA SP-506 (Noordwijk: ESA), 71

Vřsnak, B., Ruždjak, V., Zlobec, P., \& Aurass, H. 1995, Sol. Phys., 158, 311

Wagner, W. J., \& MacQueen, R. M. 1983, A\&A, 120, 136

Warmuth, A., Vrsnak, B., Aurass, H., \& Hanslmeier, A. 2001, ApJ, 560, L105

Webb, D. F., \& Howard, R. A. 1994, J. Geophys. Res., 99, 4201 